\title{
Prevalence and factors associated with intestinal parasitic infections among food handlers of Southern Ethiopia: cross sectional study
}

Mohammedaman Mama* and Getaneh Alemu

\begin{abstract}
Background: Globally about one third of the total population is estimated to be infected with intestinal parasites, of which, the majority are people living in tropical and sub-tropical parts of the world. Cases of intestinal parasitosis are also highly abundant in Ethiopia and hence the aim of present study was to assess prevalence and predictors of intestinal parasitic infections among food handlers working in Arba Minch University students' cafeteria, South Ethiopia.

Method: A cross sectional study was conducted among food handlers working in Arba Minch University from April to June, 2015. A pretested structured questionnaire was used for collecting data about socio-demographic characteristics and possible risk factors. Stool specimens were collected and examined microscopically for the presence of eggs, cysts and trophozoites of intestinal parasites. Data entry and analysis were done using SPSS version 20 software.

Results: A total of 376 food handlers were enrolled in the study of which thirty one of them were not willing to participate for a stool examination. The majority of study participants were females 273 (72.6\%). About 123 (36\%) of food handlers were found to be positive for different intestinal parasites with the most abundant parasite of Entamoeba histolytica/dispar 48 (14\%) followed by Ascaris lumbricoides 32 (9.27\%). Finger nail status (AOR: 2.2, 95 \% Cl: 1.29-3.72), hand washing practice after toilet (AOR: 1.71, $95 \%$ Cl: 1.06-2.77), hand washing practice before food handling (AOR: 1.69, 95 \% Cl: 1.04-2.75), preparing food when suffering from diseases (AOR: 3.08, 95 \% Cl: 1.17-8.13), and using common knife for cutting raw flesh food and other food (AOR: 1.72, $95 \%$ Cl: 1.01-2.92) were independent predictors of intestinal parasitic infection among the food handlers.

Conclusion: This study revealed a high prevalence of intestinal parasites among food handlers. Since most of the intestinal parasites are transmitted by the feco-oral route, food handlers could be an important source of infection to the students and general population. Therefore, constant epidemiological surveillance through biannual routine parasitological tests and treatment of the infected cases along with the improvement of personal hygiene and environmental sanitation are recommended to control the parasitic infection in food handlers.
\end{abstract}

Keywords: Intestinal parasites, Food handlers, Ethiopia

\footnotetext{
* Correspondence: muheekoo@gmail.com

Department of Medical Laboratory Science, College of Medicine and Health

Sciences, Arba Minch University, Arba Minch, Ethiopia
} 


\section{Background}

Food borne diseases are public health problems worldwide. It was estimated that about $30 \%$ of the population living in the developed world suffers from diarrheal diseases, mostly caused by food borne microbial pathogens. About 2 million deaths occur annually due to food borne diseases in developing countries [1,2].

Globally about one third of the total population is estimated to be infected with intestinal parasites, the majority being people living in tropical and sub-tropical parts of the world [3]. About 819 million people are infected with Ascaris lumbricoides (A. lumbricoides), 464.6 million people with Trichuris trichiura (T. trichuira), 438.9 million people with hookworm infection [4], 500 million people with Entamoeba histolytica (E. histolytica), and 2.8 million people are infected with Giardia lamblia (G. lamblia) [5].

As in many developing countries, cases of intestinal parasitosis are also highly abundant in Ethiopia. It is estimated that one third of Ethiopians are infected with A. lumbricoides, one quarter is infected with T. trichiura and one in eight lives with hookworm. As a result, Ethiopia has the second highest burden of ascariasis, the third highest burden of hookworm, and the fourth highest burden of trichuriasis in Sub-Saharan Africa [6].

As a result of this, the Federal Ministry of Health $(\mathrm{FMoH})$ of Ethiopia has prioritized intestinal parasitic infection as one of the Neglected Tropical Diseases (NTDs) in the National Master Plan of NTDs, to address the public health problems due to NTDs [7].

High prevalence of intestinal parasitic infections and poly parasitism affect the health status of individuals mainly affecting physical and mental developments causing malnutrition, anaemia, stunting, cognitive impairment, lowered educational achievement and interfering with productivity $[8,9]$. It was estimated and indicated by different researchers; high prevalence of intestinal parasites is largely due to lack of personal and environmental sanitation, lack of safe water supply, human behavior, poverty, ignorance of health promotion practices and impoverished health services [10-12].

Transmission of intestinal parasites is effected directly or indirectly through faeces contaminated objects such as food, water, soil and finger. Although various modes of transmission of intestinal parasites are known to exist, several studies have shown the higher magnitude of hand to mouth transmission as potential sources of exposure to parasitic infections [13, 14]. Accordingly, food handlers with poor personal hygiene working in food serving establishments could be potential sources of infections by many of the intestinal helminths and protozoa. Therefore, food handlers with poor personal hygiene working in food service establishments could be potential sources of infections $[15,16]$.
Various studies have been conducted to assess how prevalent intestinal parasitic infections among food handlers in different parts of the world including Ethiopia. In Ethiopia, the rate of infection with intestinal parasites among food handlers ranged from 29 to $72 \%$ with different reports on the predominant species of parasites and hygiene practices $[1,10,13,14]$. The importance of food handlers as threats in the transmissions of parasitic diseases has been stressed by several authors $[9,17,18]$ and therefore, the aim of this study was to determine intestinal prevalence and associated risk factors among food handlers working in Arba Minch University Students' Cafeteria.

\section{Methods}

\section{Study area, design and period}

A cross sectional study design was conducted at Arba Minch Universityfrom April- June, 2015. Arba Minch University is one of the well-established universities found in the Southern Nations, Nationalities and People's Region (SNNPR). It is located at Arba Minch town, $500 \mathrm{~km}$ south of Addis Ababa. The main campus of the university is situated at the eastern foot of Gamo mountain ranges and adjacent to the vast low land stretching towards Lake Abaya and Lake Chamo which form part of the East African Rift Valley. The spectacular features of the twin Rift Valley lakes, Abaya and Chamo, impart a picturesque view to the University as it is viewed from the main campus hills. Its two new campuses are named after these two lakes while the third new campus is named after one of the wonderful national parks of the country - Nech Sar. The gifted land of the South offers a huge opportunity to the University to venture into new territories of education, research and development. At present, the University runs both undergraduate and post graduate programs in five campuses with 17,932 total number of students which enrolled in the regular and continuing education programs.

\section{Sample size determination and sampling technique}

The sample size was determined using sample size determination for estimation of single population proportion formula. Taking $63 \%$ parasite prevalence from previous study [19], $95 \%$ confidence interval $(\mathrm{z}=1.96)$ and $5 \%$ marginal error $(\mathrm{d}=0.05)$ the initial sample size was 358 and, finally by considering a $5 \%(\approx 18$ subjects $)$ non response rate, the final sample size was determined to be 376 .

To select representative groups from 1000 number of total food handlers a proportional sample size was determined for each stratum (campuses), and food handlers were selected randomly by lottery method from the roster lists of food handlers which was obtained from cafeteria office of each Arba Minch University campuses. 


\section{Data collection and laboratory processing}

Data related to socio-demographic characteristics, and personal hygiene practices of food handlers and related risk factors was collected by face to face interview using pre tested structured questionnaire and observational guidelines. All the questionnaires were checked for accuracy and completeness. After proper instruction, food handlers were given labeled collection cups that contain applicator sticks. From each study subject, about $2 \mathrm{~g}$ of fresh stool was collected. Each of the specimens was checked for its label, quantity and procedure of collection. A portion each of the stool samples was processed with a direct microscopic technique to detect cysts, trophozoites, eggs and larva of intestinal parasites immediately. The remaining part of the samples was transported to research laboratory. Stool examinations were performed using the formol-ether concentration technique. Both the 10x and 40x objectives were used for detection of eggs and larvae of helminth and cysts and trophozoites of protozoan parasites. Iodine solution was used to detect and identify cysts of protozoan parasites.

Well experienced and trained laboratory technologist was recruited for laboratory examination. Before the actual stool specimen's examination for the study subjects, pre-test was conducted on stool samples collected from patients attending student clinic to look the reliability/ reproducibility of stool examination procedure by the laboratory technologist with two different microscopes.

\section{Data analysis}

Data was edited, cleaned, entered and analyzed using statistical package for social science (SPSS) version 20. Descriptive analysis such as frequencies and mean was used. Initially the association between each exposure and the presence of infection was assessed using the chisquare test, and odds ratio was computed to measure the strength of the association. Univariate and bivariate analysis were conducted and crude and adjusted odds ratio with $95 \%$ CI were calculated for statistical significance tests. Variables with significant at $P<0.3$ in a bivariate analysis were considered for multivariate analysis through multiple logistic regression model to look their relative effect on the outcome variable by controlling other possible confounding factors. $P$-value of $\leq 0.05$ was considered to indicate statistical association. Finally the result was presented using tables.

\section{Ethical considerations}

The study protocol was ethically approved by review boards of Arba Minch University College of Medicine and Health Sciences with project code of GOV/AMU/ TH.5-2/CMHS/MLS/02/07. Letter of cooperation was written to each leader of the cafeteria. Informed verbal consent was obtained from each study participant. Strict confidentiality was maintained during the interview process as well as anonymity was kept during data processing and report writing. Food handlers positive for intestinal parasites were treated.

\section{Results \\ Socio demographic characteristics}

A total of 376 food handlers were selected and all of them agreed to participate so that the response rate was $100 \%$. About $7.4 \%$ were aged less than 20 years and the majority (63.3\%) lay in the working age group of 21-35 years. Majority of the workers were females $(72.6 \%)$ and were educated up to primary level (36.4\%). Only $36.2 \%$ food handlers had certificate in food training and none of them had medical check-up (Table 1).

\section{Prevalence of intestinal parasites}

From the total study subjects $(n=376)$, thirty one of them were not willing to participate for a stool examination. As a result, a total of 345 food handlers

Table 1 The prevalence of intestinal parasitic infection with respect to socio-demographic characteristics of food handlers at Arba Minch University students' cafeteria, Arba Minch, South Ethiopia, April- June 2015

\begin{tabular}{|c|c|c|c|c|}
\hline \multirow[t]{2}{*}{$\begin{array}{l}\text { Demographic } \\
\text { characters }\end{array}$} & Infected & $\begin{array}{l}\text { Not } \\
\text { infected }\end{array}$ & \multirow[t]{2}{*}{$x^{2}$} & \multirow[t]{2}{*}{$p$-value } \\
\hline & No. (\%) & No. (\%) & & \\
\hline \multicolumn{5}{|l|}{ Sex } \\
\hline Male & $41(40)$ & $62(60)$ & 4.36 & 0.037 \\
\hline Female & 78 (28.6) & $195(71.4)$ & & \\
\hline \multicolumn{5}{|l|}{ Age in years } \\
\hline$\leq 20$ & $14(50)$ & $14(50)$ & 8.05 & 0.045 \\
\hline $21-35$ & $71(29.8)$ & $167(70.2)$ & & \\
\hline $36-50$ & $34(33)$ & $69(67)$ & & \\
\hline$\geq 50$ & 0 & $7(100)$ & & \\
\hline \multicolumn{5}{|l|}{ Years of service } \\
\hline$<1$ year & $15(58)$ & $11(42)$ & 11.12 & 0.011 \\
\hline $1-5$ years & $49(30)$ & $112(70)$ & & \\
\hline 6-10years & $38(32)$ & $79(68)$ & & \\
\hline$>10$ years & 17 (23.6) & $55(76.4)$ & & \\
\hline \multicolumn{5}{|l|}{ Educational status } \\
\hline Illiterate & $11(31)$ & $24(69)$ & 2.62 & 0.45 \\
\hline Primary school & $40(29.2$ & $97(70.8)$ & & \\
\hline Secondary school & $29(28.7)$ & $72(71.3)$ & & \\
\hline $\begin{array}{l}\text { Higher than secondary } \\
\text { school }\end{array}$ & $39(38)$ & $64(62)$ & & \\
\hline \multicolumn{5}{|l|}{ Certified in food training } \\
\hline No & $73(30.4)$ & $167(69.6)$ & 0.53 & 0.77 \\
\hline Yes & $46(33.8)$ & $90(66.2)$ & & \\
\hline
\end{tabular}


participated for stool examination of whom, 123 (36 \%) were found to be positive for various types of intestinal parasites. Of those infected, 66 (19.13\%) had helminthic infections, while the remaining 57 (16.52\%) had protozoan infection. The most abundant parasite was $E$. histolytica/dispar 48 (14\%) followed by A. lumbricoides 32 (9.3\%). Among 123 positive food handler's, 2(0.6\%) had mixed infections (Table 2).

\section{Intestinal parasitic infections and associated factors}

As shown in Table 3, different factors were assessed for possible association with intestinal parasitic infection among the study participants. The results of the study showed that $40 \%(41 / 103)$ of male and $28.6 \%(78 / 273)$ female participants were found to be infected with at least one parasite. The prevalence of infection with intestinal parasites have initial association with sex of the respondents $(p=0.038)$, However the association was not significant after adjusting for confounders using multivariate logistic regression $(p=0.530)$.

Food handlers aged below 20 years of age were found to have a high percentage (50\%) of infection as compared to other age groups. The association between age groups and intestinal parasitic infection was statistically significant $(p=0.034)$. After adjusting for possible confounders by multivariate logistic regression, the prevalence of infection with intestinal parasites was not significantly different among different age groups of food handlers. Similarly years of service does not have significant association with parasitic infection $(A O R=0.086)$. Forty four

Table 2 Type and prevalence of intestinal parasites isolated from stool specimens of food handlers at Arba Minch University students' cafeteria, Arba Minch, South Ethiopia, April- June 2015

\begin{tabular}{ll}
\hline Parasite Species & Frequency (\%) \\
\hline Protozoa & $66(19.13)$ \\
E. histolytica/dispar & $48(14)$ \\
Trophozoite form & $35(10.14)$ \\
Cyst form & $13(3.76)$ \\
G. lamblia & $18(5.22)$ \\
Trophozoite form & $12(3.5)$ \\
Cyst form & $5(1.44)$ \\
Helminthes & $57(16.52)$ \\
A. lumbricoides & $31(9)$ \\
Taenia species & $14(4.1)$ \\
T. trichiura & $4(1.2)$ \\
Hookworms & $3(0.9)$ \\
S. stercoralis & $2(0.6)$ \\
Mixed infection & $2(0.6)$ \\
Cyst of E. histolytica/dispar and Teania species & $1(0.3)$ \\
Ova of A. lumbricoides, T. trichiura and Hookworms & $1(0.3)$ \\
\hline
\end{tabular}

(49 \%) of food handlers with untrimmed finger nails had infection with at least one parasite (Table 3). The finger nail status of the study participants had a significant association with the rate of intestinal parasitic infection $(p=0.004)$. The odds of parasitic infection was 2 times higher (AOR: 2.193, 95 \% CI [1.29-3.72]) for food handlers who had untrimmed finger nail as compared to those who trimmed.

The practice of hand washing after toilet $(p=0.029)$ and before food handling ( $p=0.034)$ was significantly associated with parasitic infection among the study participants. Food handler's who were using water only when they washed their hands after toilet had a more likely risk of infection (with $71 \%$ ) for intestinal parasites [OR: 1.71, $95 \%$ CI; (1.057-2.765)] than food handler's who use water and soap. The extent of intestinal parasitosis was more likely to occur (with $69 \%$ ) among food handlers who washed their hands before food handling with water only [OR: 1.69, $95 \%$ CI (1.04-2.75)] than food handlers who wash their hand with water and soap (Table 4).

Preparing food when suffering from disease like diarrhea, skin disease had a significant association with the rate of intestinal parasitic infection $(p=0.023)$. The odds of parasitic infection was 3 times higher (AOR: 3.077, $95 \%$ CI [1.165-8.127]) for individuals who had disease and participate on food preparation as compared to those who did not prepare food when they are diseased. The practice of using common knife for cutting raw flesh food and other food had a statistically significant association with intestinal parasitic infection $(p=0.046)$ (Table 4$)$. The multivariate logistic regression model estimated that individuals who use common knife for cutting raw flesh food and other food were $72 \%$ times (AOR: 1.72, $95 \%$ CI: 1.01-2.92) more likely to be infected with intestinal parasites than those who did not use common knife for cutting raw flesh food and other food.

\section{Discussion}

Food handlers may be carrying a wide range of intestinal parasites and have been implicated in the transmission of many infections to the public in the community and to students in University. The spread of disease via food handlers is a common and persistent problem worldwide $[13,20]$. Therefore, this study was undertaken to assess prevalence of intestinal parasites, among food handlers of Arba Minch University Student's Cafeteria, Arba Minch, South Ethiopia.

In this study, most of food handlers working in students' cafeteria of Arba Minch University were females, young adults and had low educational levels; which is in line with studies from different parts of Ethiopia like Bahir Dar, Gondar town and Addis Ababa [13, 14, 21]. The overall proportion of infected female food handlers 
Table 3 Multivariate analysis of intestinal parasitic infections and potential risk factors among food handlers at Arba Minch University Students Cafeteria, Arba Minch, South Ethiopia, April- June 2015

\begin{tabular}{|c|c|c|c|c|c|c|}
\hline \multirow[t]{2}{*}{ Variables } & Negative & Positive & \multirow{2}{*}{$\begin{array}{l}\text { Crude OR } \\
(95 \% \mathrm{Cl})\end{array}$} & \multirow{2}{*}{$\begin{array}{l}P \text { - } \\
\text { value }\end{array}$} & \multirow{2}{*}{$\begin{array}{l}\text { Adjusted OR } \\
(95 \% \mathrm{Cl})\end{array}$} & \multirow[t]{2}{*}{$P$-value } \\
\hline & No. (\%) & No. (\%) & & & & \\
\hline \multicolumn{7}{|l|}{ Sex } \\
\hline Male & $62(60)$ & $41(40)$ & $1.653(1.029-2.656)$ & 0.038 & $1.199(0.680-2.114)$ & 0.530 \\
\hline Female & 195(71.4) & 78(28.6) & 1.00 & & 1.00 & \\
\hline \multicolumn{7}{|l|}{ Age } \\
\hline$<20$ years & $14(50)$ & $14(50)$ & 2.352(1.066-5.188), & 0.034 & 1.355(0.523-3.509) & 0.531 \\
\hline $21-35$ years & 167(70.2) & $71(29.8)$ & 1.00 & & 1.00 & \\
\hline $36-50$ years & $69(67)$ & $34(33)$ & $1.159(0.706-1.903)$ & 0.560 & $1.162(0.664-2.033)$ & 0.599 \\
\hline$>50$ years & $7(100)$ & 0 & 0.000 & 0.999 & 0.000 & 0.999 \\
\hline \multicolumn{7}{|l|}{ Years of Service } \\
\hline$<1$ year & $11(42)$ & $15(58)$ & $3.117(1.336-7.273)$ & 0.009 & $2.320(0.887-6.068)$ & 0.086 \\
\hline $1-5$ years & $112(70)$ & 49(30) & 1.00 & & 1.00 & \\
\hline $6-10$ years & $79(68)$ & $38(32)$ & 1.099(0.659-1.835) & 0.717 & $1.298(0.724-2.329)$ & 0.381 \\
\hline$>10$ years & $55(76.4)$ & 17(23.6) & 0.706(0.373-1.339) & 0.287 & $.739(0.351-1.557)$ & 0.426 \\
\hline \multicolumn{7}{|l|}{ Finger nail Status } \\
\hline Not trimmed & $46(51)$ & $44(49)$ & $2.691(1.648-4.393)$ & 0.000 & $2.193(1.293-3.719)$ & 0.004 \\
\hline Trimmed & $211(74)$ & $75(26)$ & 1.00 & & 1.00 & \\
\hline
\end{tabular}

$(22.6 \%)$ with intestinal parasites was higher than the proportion of infected male food handlers (12\%). This can be due to the fact that women are much more involved in kitchen work than men. Most of the males participate in the delivery of the already prepared food, while women are those who go bare footed during the preparation of the food, as well as those who do the washing of vegetables and fruits mainly in the kitchen.

Concerning the relation of age group and parasitic infection, the study revealed relatively a higher infection rate in the age group younger than 20 years. No significant difference was found in the distribution of parasitic infection among all age groups which shows that there is equal exposure to the infection and suggests an effect of environmental conditions on infection.

It is expected that all food handlers at University, military, hospitals etc. cafeterias to have a periodic medical checkup for food borne pathogens. Despite this fact, the interview result of our study showed that none of food handlers had a medical checkup for intestinal infection

Table 4 Multivariate analysis of intestinal parasitic infections and potential hygiene risk factors among food handlers at Arba Minch University Students Cafeteria, Arba Minch, South Ethiopia, April- June 2015

\begin{tabular}{|c|c|c|c|c|c|c|}
\hline \multirow[t]{2}{*}{ Variables } & Negative & Positive & \multirow{2}{*}{$\begin{array}{l}\text { Crude OR } \\
(95 \% \text { Cl) }\end{array}$} & \multirow[t]{2}{*}{$P$-value } & \multirow{2}{*}{$\begin{array}{l}\text { Adjusted OR } \\
(95 \% \mathrm{Cl})\end{array}$} & \multirow[t]{2}{*}{$P$-value } \\
\hline & No. (\%) & No. (\%) & & & & \\
\hline \multicolumn{7}{|l|}{ Hand washing after toilet } \\
\hline With water only & $100(61)$ & 64(39) & $1.827(1.178-2.834)$ & 0.007 & $1.710(1.057-2.765)$ & 0.029 \\
\hline With water and soap & 157(74) & $55(26)$ & 1.00 & & 1.00 & \\
\hline \multicolumn{7}{|c|}{ Hand washing before food handling } \\
\hline With water only & $125(63)$ & $74(27)$ & $1.790(1.142-2.804)$ & 0.011 & $1.691(1.040-2.749)$ & 0.034 \\
\hline With water and soap & $130(75)$ & $43(25)$ & 1.00 & & 1.00 & \\
\hline \multicolumn{7}{|c|}{ Preparing food when suffering from diseases like diarrhea, cold or skin diseases } \\
\hline No & $33(85)$ & $6(15)$ & 1.00 & & 1.00 & \\
\hline Yes & $224(66.5)$ & 113(33.5) & $0.360(0.147-0.885)$ & 0.026 & $3.077(1.165-8.127)$ & 0.023 \\
\hline \multicolumn{7}{|c|}{ Using common knife for cutting raw flesh food and other food } \\
\hline No & $97(74)$ & 29(26) & 1.00 & & 1.00 & \\
\hline Yes & 160(64) & $90(36)$ & $0.532(0.326-0.866)$ & 0.011 & $1.715(1.008-2.917)$ & 0.046 \\
\hline
\end{tabular}


which agrees with study done in Bahir Dar town, Ethiopia [14].

In this study about $36 \%$ of the food handlers were carriers of one or more of the pathogenic intestinal parasites. This is comparable with the finding of $38.1 \%$ done in Nigeria [22], $32 \%$ in Makka, Saudi Arabia [23] and $41.1 \%$ in Bahir dar, Northwest Ethiopia [14]. However, it is higher than study of 1.3 to $7 \%$ in India [16], $6.9 \%$ in Omdurman, Sudan [24], $8.8 \%$ in Turkey [25], $15.5 \%$ in Sari, Northern Iran [11], 24.3 \% in Gaza Strip, Palestine [26] and $29.1 \%$ in Gondar, Northwest Ethiopia [21]. It was lower than the prevalence of $44.1 \%$ from Jimma [1], $45.3 \%$ from Addis Ababa [13], 49.4 \% from Mekelle [10], 58.4 \% from Jimma [9] and $71.8 \%$ from Addis Ababa, Ethiopia [27]. The differences in reported prevalence in various studies may be due to socioeconomic status, climatic conditions, poverty, and personal and community hygiene.

In the present study, multiple intestinal parasitic infections were found with $A$. lumbricoides being the predominant parasite from helminthes followed by Taenia species, and E. histolytica/dispar was the most predominant from protozoan parasites followed by G. lamblia. High prevalence of ascariasis is a good indicator of improper fecal disposal, while that of entameobiasis reflects use of poor water quality among the study participants. This was consistent with the finding of a similar study conducted in Enugu State, Nigeria [28], and in different parts of Ethiopia [9, 10, 13, 14].

In this study encouraging results were obtained regarding practices of hand washing after toilet and before food preparation. Food handlers' hand washing practices after toilet $(100 \%)$ was in parallel with the previous studies done in Ethiopia like, $90.6 \%$ in Bahir dar [14]. However, hand washing practices of food handlers was low especially after touching body parts and after blowing nose which increase the likely hood of microorganisms cross contamination. These reflected that food handlers lack awareness about food contamination with poor hygienic practices.

Poor personal hygiene, including inadequate hand washing among food handlers is a common practice that contributes to food born diseases [10]. Parasite eggs in the soil can contaminate vegetables, then hands and hence directly enter into the mouth, or ingested by eating raw vegetables $[11,29]$.

This study identified high protozoan and helminthic infection that can easily be transmitted via feco-oral route, either directly from person to person or indirectly by eating or drinking fecally contaminated food and water. Hence, in this study multivariate logistic regression model indicated that untrimmed finger nail, hand washing practice after toilet, hand washing before food handling, preparing food when suffering from diseases like diarrhea and using common knife for cutting raw flesh food and other food were identified as determinant factors for food handlers being infected by intestinal parasites. The present study was subjected to the following limitations. The study was non-blinded. Due to lack of antigen tests, Entamoeba histolytica and Entamoeba dispar were not separated. This study did not attempt to assess the parasite carriage of the finger nail contents and parasite intensity due to logistic reasons. Specific methods such as the adhesive scotch tape for E. vermicularis, Harada Moori's filter Paper for S. stercoralis and for hookworm infections was not done.

\section{Conclusion}

This study revealed a high prevalence of intestinal parasites among food handlers. Since most of the intestinal parasites are transmitted by the feco-oral route, food handlers could be an important source of infection to the general population. Therefore, constant epidemiological surveillance through biannual routine parasitological tests and treatment of the infected cases along with the improvement of personal hygiene and environmental sanitation are recommended to control the parasitic infection in food handlers.

\section{Competing interests \\ The authors declare that they have no competing interests.}

\section{Authors' contributions}

MM: Primary researcher and conceived the idea for this study. MM and GA: Participated in data collection, conducted data analysis, drafted and finalized the manuscript for publication. Both authors read and approved the final manuscript.

\section{Acknowledgments}

We would like to thank Arba Minch University, College of Medicine and health Sciences for funding this research. A very special thanks to ethical review boards of Arba Minch University for giving ethical clearance. We are also indebted to the study participants and data collectors.

Received: 16 September 2015 Accepted: 27 January 2016 Published online: 01 February 2016

\section{References}

1. Tefera T, Mebrie G. Prevalence and predictors of intestinal parasites among food handlers in Yebu town, southwest Ethiopia. PLoS One. 2014;9:e110621. WHO. Food Safety and Foodborne IIIness. Fact sheet No 237. 2007.

3. Chan M-S. The global burden of intestinal nematode infections - fifty years on. Parasitol Today. 1997;13(11):438-43

4. Pullan RL, Smith JL, Jasrasaria R, Brooker SJ. Global numbers of infection and disease burden of soil transmitted helminth infections in 2010. Parasit Vectors. 2014:7(1):37.

5. Duc PP, Nguyen-Viet H, Hattendorf J, Zinsstag J, Cam PD, Odermatt P. Risk factors for Entamoeba histolytica infection in an agricultural community in Hanam province, Vietnam. Parasit Vectors. 2011;4:102-11.

6. Deribe K, Meribo K, Gebre T, Hailu A, Ali A, Aseffa A, et al. The burden of neglected tropical diseases in Ethiopia, and opportunities for integrated control and elimination. Parasit Vectors. 2012;5(1):240.

7. FMoH. National Master plan for Neglected trpical Diseases (NTDS) 2013-2015. Addis Ababa: FMoH; 2013.

8. Guyatt H. Do intestinal nematodes affect productivity in adulthood? Parasitol Today. 2000;16(4):153-8.

9. Sahlemariam Z, Mekete G. Examination of finger nail contents and stool for ova, cyst and larva of intestinal parasites from food handlers working in 
student cafeterias in three higher institutions in Jimma. Ethiopian J Health Sci. 2001;11(2):131-7.

10. Nigusse D, Kumie A. Food hygiene practices and prevalence of intestinal parasites among food handlers working in Mekelle university student's cafeteria, Mekelle. GARJSS. 2012;1(4):65-71.

11. Sharif M, Daryani A, Kia E, Rezaei F, Nasiri M, Nasrolahei M. Prevalence of intestinal parasites among food handlers of Sari, Northern Iran. Rev Inst Med Trop Sao Paulo. 2015;57(2):139-44.

12. Wakid MH, Azhar El, Zafar TA. Intestinal parasitic infection among food handlers in the holy city of Makkah during Hajj season JKAU. Med Sci. 2009:16(1):39-52.

13. Aklilu A, Kahase D, Dessalegn M, Tarekegn N, Gebremichael S, Zenebe S, et al. Prevalence of intestinal parasites, salmonella and shigella among apparently health food handlers of Addis Ababa University student's cafeteria, Addis Ababa, Ethiopia. BMC Res Notes. 2015:8(1):17.

14. Abera B, Biadegelgen F, Bezabih B. Prevalence of Salmonella typhi and intestinal parasites among food handlers in Bahir Dar Town, Northwest Ethiopia. Ethiop J Health Dev. 2010;24(1):46-50.

15. Organization WH. Health surveillance and management procedures for food-handling personnel: report of a WHO consultation [held in Geneva from 18 to 22 April 1988]. 1989.

16. Khurana S, Taneja N, Thapar R, Sharma M, Malla N. Intestinal bacterial and parasitic infections among food handlers in a tertiary care hospital of North India. Trop Gastroenterol. 2010;29(4):207-9.

17. Tsega E, Nadew F. The threat of ameobic cyst carriers among hospital food handlers. Ethio Med J. 1972;10:43-53.

18. Dagnew M, Tiruneh M, Moges F, Tekeste Z. Survey of nasal carriage of Staphylococcus aureus and intestinal parasites among food handlers working at Gondar University, Northwest Ethiopia. BMC Public Health. 2012;12(1):837.

19. Teklemarium S, Roma B, Sorsa S, Worku S, Erosie L. Assessment of sanitary and hygienic status of catering establishments of Awassa Town. Ethiop J Health Dev. 2000;14(1):91-8.

20. MohanV DL, Lal M. An evaluation of health status of food handlers of eating establishments in various educational and health institutions in Amritsar. Indian J comm Med. 2001;26(2):80-5.

21. Andargie G, Kassu A, Moges F, Tiruneh M, Huruy K. Prevalence of bacteria and intestinal parasites among food-handlers in Gondar town, northwest Ethiopia. J Health Popul Nutr. 2008:26(4):451.

22. Ifeadike C, Ironkwe O, Adogu P, Nnebue C, Emelumadu O, Nwabueze S, et al. Prevalence and pattern of bacteria and intestinal parasites among food handlers in the Federal Capital Territory of Nigeria. Niger Med J. 2012;53(3):166

23. Majed H, Wakid EIA, Zafar A. Intestinal parasitic infection among food handlers in the holy city of Makkah during Hajj season 1428 hegira (2007G). Med Sci. 2009;16(1):39-52.

24. Saeed HA, Hamid HH. Bacteriological and parasitological assessment of food handlers in the Omdurman area of Sudan. J Microbiol Immunol Infect. 2010;43(1):70-3.

25. Gunduz T, Limoncu M, Cumen S, Ari A, Etiz S, Tay Z. Prevalence of intestinal parasites and nasal carriage of Staphylococcus areus among food handlers in Manisa Turky. J Environ Health. 2008;18(5):230-5.

26. Ashour N, Hassan I. Occurrence of Intestinal Parasites and Hygiene Characters among Food Handlers in Gaza Strip, Palestine. Annals of Alquds Medicine. 2012:8:2-13.

27. Mekonnen B, Erko B, Legesse M. Prevalence of intestinal parasitic infections and related risk factors among street dwellers in Addis Ababa. Ethiopia Trop Dis. 2014;2(132):2

28. Egbuobi R, Nwagbaraocha M, Dike-Ndudim J, Okorie H, Ogamaka I, Egbuobi $L$, et al. Incidence of intestinal parasites among food handlers (hawkers) around the University of Nigeria teaching hospital Enuqu, Enugu state, Nigeria. Open J Med Microbiol. 2014;4:23-8.

29. Ulukanligil M, Seyrek A, Aslan G, Ozbilge H, Atay S. Environmental pollution with soil-transmitted helminths in Sanliurfa, Turkey. Mem Inst Oswaldo Cruz. 2001:96(7):903-9.

\section{Submit your next manuscript to BioMed Central and we will help you at every step:}

- We accept pre-submission inquiries

- Our selector tool helps you to find the most relevant journal

- We provide round the clock customer support

- Convenient online submission

- Thorough peer review

- Inclusion in PubMed and all major indexing services

- Maximum visibility for your research

Submit your manuscript at www.biomedcentral.com/submit
Biomed Central 